\title{
Effects of sex and strain on exploratory locomotion and development of nonreinforced maze patrolling
}

\author{
K. BÄTTIG and J. SCHLATTER \\ Swiss Federal Institute of Technology, 8092 Zurich, Switzerland
}

\begin{abstract}
Continuous recording of locomotion in an enclosed maze with no reinforcement other than that intrinsic to ambulation through the maze provided evidence for a dissociation between the efficiency of patrolling the entire maze and locomotor activity. Patrolling efficiency improved with repeated exposure to a single maze or to two similar maze configurations but not with daily presentation of dissimilar mazes. There were only small strain and sex differences in the development of efficient patrolling, whereas locomotor activity was considerably higher in female Roman high-avoidance (RHA) rats than in female Roman low-avoidance rats and higher in RHA female rats than in RHA male rats.
\end{abstract}

Ambulation of rodents through complex mazes appears to the observer as rather erratic behavior. Pauses and changes of direction seem to occur in a more or less unpredictable sequence. The fact that the overall amount of locomotion itself is influenced by the novelty and stimulus complexity of the situation (Berlyne, 1950; Bindra \& Spinner, 1958) has led to the widespread interpretation of such locomotion as "exploratory behavior." This interpretation, which is especially popular in psychopharmalogical studies, represents an overgeneralization, as shown by Lore (1968). He reviewed ample data suggesting that a specific locomotor drive, in addition to the stimulus content of the environment, accounted for spontaneous locomotion.

Separation of the exploratory and locomotor components of ambulation within a maze has been attempted in several studies (Corman \& Shafer, 1968; Kumar, 1969; Leyland, Robbins, \& Iversen, 1976; Robbins \& Iversen, 1973). These studies used open or enclosed fields, runways, or Y-mazes, into which distinctive stimuli were introduced. Locomotion was recorded by photocells, while exploration was evaluated by the duration of preoccupation of an animal with the distinctive stimulus. Latent learning, as evidenced by stimulus habituation or facilitation of learning in subsequent specific tasks, was observed but was not found to be correlated with the amount of locomotion. This approach, however, neglected

\footnotetext{
This study was supported by Grant $3.160-0.73$ from the "Swiss National Science Foundation." The authors also thank Dr. James R. Martin, for editorial assistance and comments in the preparation of this manuscript, and Dr. P. Driscoll, for the careful maintenance of the separate RHA and RLA strains. Requests for reprints should be sent to Professor K. Bättig, Department of Behavioral Sciences, Swiss Federal Institute of Technology, Turnerstrasse 1, 8092 Zurich, Switzerland.
}

the spatial information obtained by the animal as it moved through the test environment. By omitting a specific stimulus object and considering a given maze structure itseif as the specific stimulus to be explored, it might be expected that the qualitative locomotor patterns would change as a result of information accumulated by the exploring animal. Such an approach necessitates continuous and detailed recording of all quantitative as well as qualitative directional aspects of maze locomotion. Barnett and Cowan (1976), using a plus-shaped living maze, found rats to preferentially enter maze arms which had not been entered recently. This tendency toward nonrepetitive choices parallels the phenomenon of spontaneous alternation reviewed by Dember (1961).

Uster, Bättig, and Nägeli (1976) confronted the rats with daily changing mazes consisting of larger or smaller hexagonal alleys, with or without additional blind radial alleys extending inward or outward from the hexagonal alley. The animals moved more rapidly through straight alley sections than through corners and choice points, and thus increased their activity with increasing alley length and decreased it with increasing structural complexity, suggesting that the exploration necessitated appropriate slowing of locomotion at points which represented the decisive cues of a complex maze structure. Although the mazes were changed daily, a gradual reduction in the initially dominant centrifugal tendency occurred. In contrast, locomotor activity remained fairly constant from day to day. These observations were later confirmed by Bättig, Driscoll, Schlatter, and Uster (1976) and extended by the demonstration of considerable quantitative, but not qualitative, behavioral differences between female Roman high-avoidance (RHA) and Roman low-avoidance (RLA) rats.

The two experiments of the present study were 
designed in order to investigate the importance of maze structure, psychogenetic background, and sex. The set of the behavioral variables extracted from the continuous locomotion records was extended by "inspection time" and "inspection activity." These variables represent time and number of places passed until an animal has reached every accessible place of a maze. These inspection variables would be expected to decrease with experience only if the animals developed a concept of the spatial structure of a maze due to an innate tendency to avoid recently visited places as evidenced by spontaneous alternation.

The first experiment compared female RHA with male RHA rats for the set of mazes used in the study of Uster et al. (1976) and a single asymmetric maze which was rotated daily by $60^{\circ}$ to eliminate the influence of extramaze cues on the maze exploration of the rat. The second experiment compared females of the Roman strains (RHA and RLA) for two asymmetric mazes. The rats were tested in only one of the two mazes or in both mazes on alternating days.

\section{EXPERIMENT 1}

\section{Method}

Subjects. The subjects, 18 male and 18 female naive RHA rats, were 5 months old at the beginning of the experiment. They were housed three per Macrolon cage in animal quarters which were illuminated from 1900 to $0700 \mathrm{~h}$. Each day during the last 10 preexperimental days, the rats were habituated to the experimental procedure. Food and water were available ad lib.

Apparatus. The ground plan of the mazes used in the experiment is shown in Figure 1. Configuration A represents the basic maze which was divided by barriers to produce the configurations 1 through 7. The enclosed alleys of the mazes were $8 \mathrm{~cm}$ wide and $15 \mathrm{~cm}$ high, and their entire interior covered with dark gray PVC-linings. Walls and ceilings were fitted together and could be lifted from the maze floor. The animals were placed into the maze through one of three openings in the ceiling, with removable covers, that provided access to one of the three hexagonal alleys. Photocells were placed into the walls of the alleys at equal distances of about $20 \mathrm{~cm}$. This amounted to 18 photocells for the outer, 12 photocells for the middle, 6 photocells for the inner hexagonal alley and to 1 photocell for each of the 12 radial
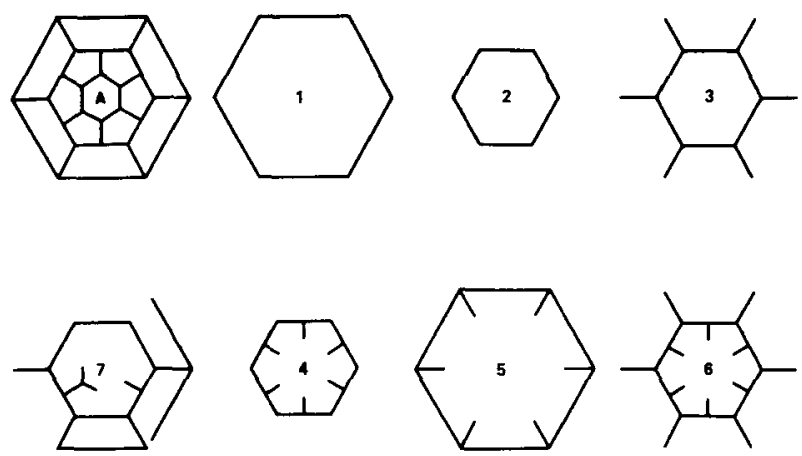

Figure 1. Ground plan of the complete hexagonal maze. 1-6: alley configurations for the daily-varied mazes. 7: alley configuration for the daily-rotated maze.

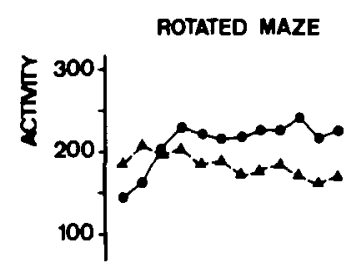

VARIED MAZES
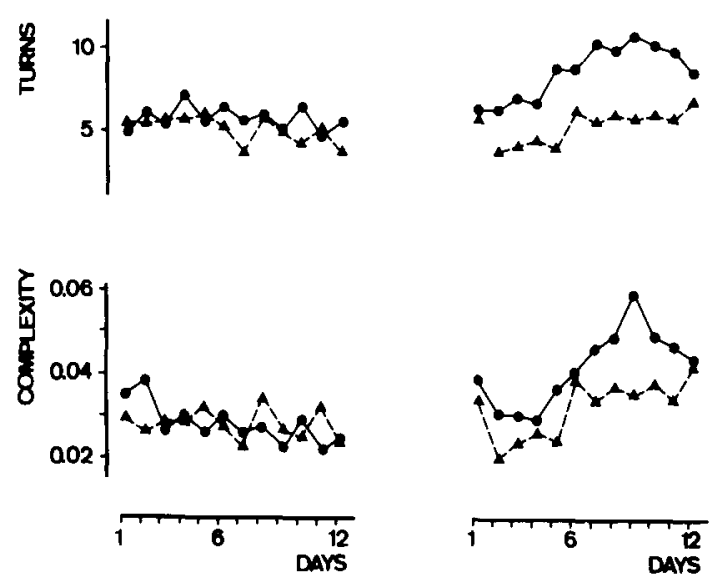

- RHA ${ }^{\circ}$ a RHÁ́

Figure 2. Comparison of all groups with regard to activity, $U$ turns and locomotor complexity over 12 testing days.

alleys. The actual location of an animal was punched on tape at a frequency of 5 cps.

Procedure. The animals were brought individually to the testing room $35 \mathrm{~min}$ before testing and returned to the home quarters immediately after testing. Testing consisted of placing an animal into the maze and recording its locomotion during $6 \mathrm{~min}$. After testing, the walls and ceilings were lifted up, the animal was removed from the floor, and the floor was wiped off with a wet sponge. The animals of each sex were divided into two experimental groups. One group of each sex was exposed on 12 successive days to the asymmetric configuration (No. 7 represented in Figure 1), which was rotated by $60^{\circ}$ at the beginning of each new testing day. The other group was exposed to mazes (Mazes 1-6) which were varied daily and presented in an individually balanced sequence during the first 6 experimental days, the same sequence being repeated during the remaining 6 days of the experiment. The intraday individual sequence of testing was varied within both groups in a balanced order to exclude possible circadian effects. Testing started $2 \mathrm{~h}$ after the beginning of the daily dark period and was terminated $2 \mathrm{~h}$ before the onset of the illumination period.

\section{Results}

Results were analyzed by processing the punched data on a Control Data Corporation computer (CDC-6000). The variables calculated were submitted to intergroup analysis of variance. Between-group differences were evaluated with $t$ tests (Sachs, 1974).

Figure 2 represents the daily averages of activity, $\mathrm{U}$ turns, and locomotor complexity. Activity was defined as the total number of photocell interruptions per testing session, $U$ turns as the number of interruptions of three adjacent photobeams twice in reversed order, and locomotor complexity as the 
number of $U$ turns divided by the activity score. Activity differed between the sexes $(p<.01)$ but not between the two maze conditions. After similar initial values for both sexes, activity in the males showed an increase but then declined slowly after the 2 nd day, whereas it continued to increase in the females until the 4th or 5th day. The overall sex difference of activity was highly significant $(p<.01)$. The number of $U$ turns remained relatively stable and was about equal for the males with the mazes daily varied and for both sexes for the repeated maze presentations. Increasing numbers of $U$ turns, however, developed in the females with the dailyvaried mazes producing a highly significant intersex difference for this testing condition $(p<.01)$. The differences seen for locomotor complexity were parallel to the intersex differences of $U$ turns. Intrasession activity decrease calculated as the regression coefficients over the four successive 90 -sec blocks of each trial did not differ between maze conditions and sex.

Figure 3 shows inspection time and inspection activity. Inspection time was defined as the time in seconds occurring until an animal had passed each accessible photocell at least once, and inspection
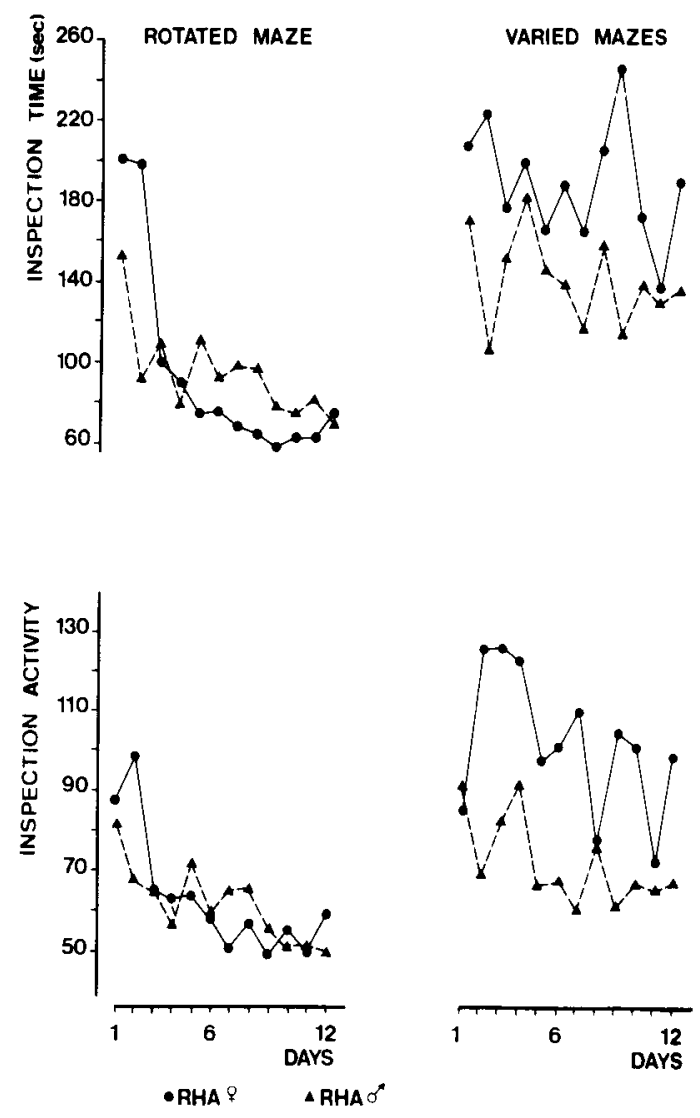

Figure 3. Comparison of all groups with regard to the patrolling behaviors (inspection time, inspection activity) over the 12 testing days. activity as the total number of photocell interruptions until this criterion had been reached. Both measures decreased regularly with the repeated mazes, but remained erratic with only modest or no improvement over the days of testing with the daily-varied mazes. In this case, the females attained significantly poorer scores than the males $(p<.01)$. With the daily-repeated maze, the only intersex difference obtained was the shorter inspection times of the females than the males during the second week $(p<.01)$, a finding due to their higher activity and locomotor speed.

A topological analysis compared these scores between different sections of the daily-repeated asymmetric mazes and between the different mazes presented to the groups tested in the daily-varied mazes. A rather pronounced pattern of topological preference developed with the asymmetric dailyrepeated maze. In comparing different maze areas, activity was calculated as an average activity per unit of alley length. The highest activity was found for the outward leading bifurcated radial alley, which was significantly higher than the activity in the hexagonal circular alley and the loop extending from it $(p<.01)$, which was in turn significantly greater than that in the outward-leading blind radial alley $(p<.01)$. The activity in both inward-leading radial alleys was significantly lower than that observed in all other maze areas $(\mathrm{p}<.01)$.

With the daily-varied mazes, the intermaze comparison revealed significant activity increases with increasing alley length. [Maze 1 vs. Maze $2(p<.05)$ and Maze 4 vs. Maze 5] and significant activity decreases for increases of complexity in terms of the number of corners and choice points [Maze 1 vs. Maze 3, 1 vs. $4(p<.05)$ and 5 vs. 6]. No sex interactions were seen in this respect with either maze condition. The frequency of leaving the hexagonal alley and entering a side alley was significantly higher for the males than for the females under both maze conditions.

\section{Discussion}

The main result of this experiment can be seen in the sex-dependent development of activity and in the inspection-related measures for the two testing procedures. Inspection was defined, in this study, as entering every accessible place of the maze. Berlyne (1960) has used the same term for the apparent preoccupation of an animal with a distinct and local stimulus. With the present experiment, however, the composite set of stimuli representing the spatial structure of an entire maze was considered as the decisive stimulus.

Efficient inspection of a maze with a minimum of unnecessary repetitive activity requires a sufficient internal representation of the maze structure which has to be acquired through an adequate cognitive 
process, suggesting latent learning. The results obtained with the asymmetric and daily-rotated maze demonstrated such latent learning by successive diminution of time and activity spent for one inspection of the entire maze. This performance appeared to be largely independent of the activity scores. Although the females were consistently more active than the males, both sexes were similar with respect to their inspection efficiency. The improvement of inspection efficiency with repeated exposure to the same maze configuration provides evidence for latent learning. This interpretation was further supported by the failure of animals to improve their performance when exposed daily to dissimilar mazes. Since these mazes were changed daily, a positive transfer of latent learning could not be expected, unless the animals acquired a general concept of all possible maze arrangements-a very difficult problem when dissimilar maze configurations were presented daily. Although a slight trend towards a continuous improvement of inspection was suggested by the respective day-to-day curves, the data remained too erratic to be conclusive. There appeared to be a pronounced sex difference that was absent in tests with the rotated asymmetric maze. The females exhibited significantly more inspection activity and required more time to enter each maze section than the males. This has been shown to be a consequence of the lower probability found for females than for males of leaving the hexagonal alley and entering side alleys.

A more rapid development of spatial latent learning than seen in the present experiment has recently been shown by Olton and Samuelson (1976). These authors used a maze consisting of a central platform with eight elevated radial paths, each leading to a food cup, and found that the rats were able to choose more than seven different arms in the course of eight alley choices after only a short period of maze habituation. They further showed that intramaze cues were not necessary for this performance. The present experimental condition differed from that of Olton and Samuelson, not only in the different maze structures used and the absence of prior adaptation and food reward, but also in the complete elimination of all extramaze cues through the use of a completely enclosed system of alleys and by daily rotation of the maze arrangement in the case of the asymmetric maze. Therefore formation of spatial concepts can be assumed to develop on the basis of different stimuli in the present study and in that of Olton and Samuelson.

Similar dissociations of "exploration and activity" have already been described by several authors using the "new object in a familiar arena" method originally proposed by Berlyne (1955). Foshee, Vierck, Meier, and Federspiel (1965) and Leyland et al.
(1976) found that the preoccupation of a rat with a novel object diminished with successive exposures, while locomotion in the arena remained quantitatively unchanged. Robbins and Iversen (1973) demonstrated further that activity and exploration were differentially susceptible to pharmacological interventions. Habituations to novel objects in a familiar arena can be expected because the objects are of no significance for the orientation and behavior of an animal. In contrast, the corners and bifurcations of a maze presumably represent the decisive orienting cues. In confirmation of an earlier study by Uster et al. (1976) using the same set of daily-varied mazes, the transition times for passing from one photocell to another were found to increase from straight sections to corners, from corners to side-alley entrance, and finally from these to exits from the side alleys. However, these relations were found to be fairly constant throughout the entire testing period, where in the Uster et al. (1976) study the longest latencies at leaving the radial alleys tended to increase with progressive experience. No obvious explanation for this difference has been found in the present data. In all other aspects, however, the present results were found to be in good agreement with the results of earlier work. Activity increased with increasing alley length and decreased with increasing geometrical complexity, and the initially strong centrifugal tendency diminished gradually as the number of test sessions increased.

\section{EXPERIMENT 2}

This experiment was carried out in order to obtain additional information about transfer of spatial latent learning from one to another maze structure. To accomplish this, both mazes contained the same radial alleys. These were accessible as centripetal alleys leading inside from the other hexagonal alley in one case and as centrifugal alleys leading outside from the other hexagonal alley in the other case. Based on the previously described preference for outward as opposed to inward leading alleys (Uster et al., 1976), it was expected that the two mazes would induce significantly different behavior. Two groups of female RHA and RLA (Roman high and low avoidance) rats were confronted with each maze on alternate days, while two other RHA groups serving as controls were assigned to continuous presentation of only one of the two mazes.

\section{Method}

Subjects. The subjects, 24 female 4-month-old RHA rats and 8 female RLA rats of the same age were maintained and tested under the same conditions as the subjects of Experiment 1.

Apparatus. The two maze configurations used are shown in Figure 4. The two maze arrangements were designed as centripetal (CP) or centrifugal (CF) according to the spatial arrangement of the radial alleys. 

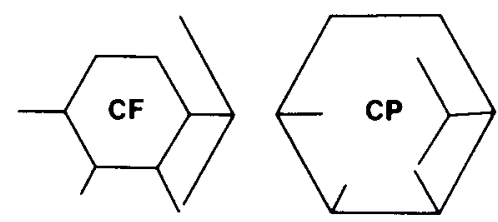

Figure 4. Alley configurations. CF: maze arrangement with outward leading radial alleys (centrifugal). CP: maze arrangement with inward leading radial alleys (centripetal).

Procedure. The daily testing procedure was the same as in Experiment 1. Each animal was tested on 6 successive days. The mazes were rotated by $60^{\circ}$ each day, and each testing session lasted $6 \mathrm{~min}$ as in the first experiment. The $24 \mathrm{RHA}$ rats were divided into three equal groups. A first group was tested in the centripetal maze only (CP), a second group in the centrifugal maze only (CF), and a third group was tested in both mazes but on alternate days (CP/CF). The 8 RLA rats were tested with both mazes on alternate days (CP/CF) only.

\section{Results}

The daily averages of activity, $U$ turns, and locomotor complexity are shown in Figure 5 . Activity increased with the three RHA CP, CF, CP/CF groups from day, but remained unchanged at a significantly lower level for the RLA CP/CF group $(p<.01)$. There was no significant difference between the RHA groups exposed to only the $\mathrm{CP}$ or the CF maze. The RHA rats tested alternately in both mazes were consistently more active on their $\mathrm{CP}$ days than on their CF days. In contrast, the activity level of the RLA CP/CF group did not change with the daily change of the mazes. The number of $U$ turns was significantly greater for the $\mathrm{CP}$ than for the $\mathrm{CF}$ mazes under all experimental conditions and in both strains $(p<.01)$, but daily alternation of the two mazes induced greater variation of this measure in the RLA than in the RHA groups. As a result, locomotor complexity, which was also greater in the RHA CP group than in the RHA CF group, showed no alterations with the alternating $\mathrm{CP} / \mathrm{CF}$ condition in the RHA rats, but varied considerably in the RLA rats. The intrasession activity decrease was consistently greater with all $\mathrm{CP}$ than $\mathrm{CF}$ conditions and with the RHA than with the RLA rats.

Figure 6 shows longer inspection times for the CP than for the CF condition in the RHA strain and for the RLA CP/CF group than for the RHA CP/CF group. For inspection activity, the picture was similar, but the RLA CP/CF group's oscillating only modestly between the two mazes was superior to that of the RHA CP/CF group in the CP maze and inferior in the CF maze so that the overall scores did not differ between the two strains. It is important to note, however, that both scores improved considerably from day to day with all conditions, including the alternated mazes.
The topological analysis revealed that all side alleys were entered significantly more often in the CF than in the CP mazes, as indicated both by the corresponding activity per unit of alley length $(p<.01)$ and decision frequencies at the choice points $(p<.01)$. The RHA CP rats developed slightly more activity per photocell in the circular hexagonal alley than did the RHA CF rats. The same difference became highly significant with the daily alternating condition of the RHA CF/CP group $(p<.01)$ The corresponding RLA CF/CP group, however, failed to show such a difference. In comparing the different side alleys, it was found that activity for all groups was significantly greater in the bifurcated side alley than in all other alleys $(p<.01)$.

The choice frequencies of entering side alleys remained nearly constant with all CF conditions. With all CP conditions, the choice frequencies were initially considerably lower than with the CF con-

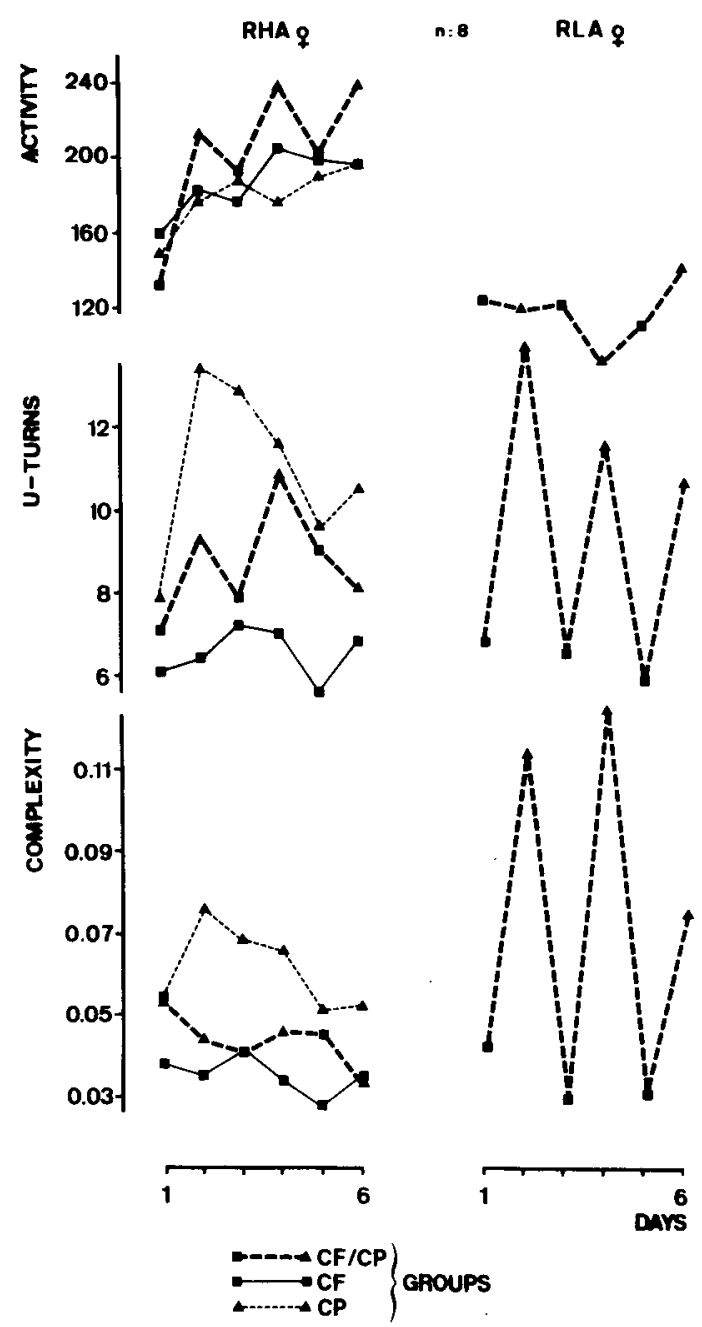

Figure 5. Comparison of all groups with regard to activity, $U$ turns and locomotor complexity over the 6 testing days. 

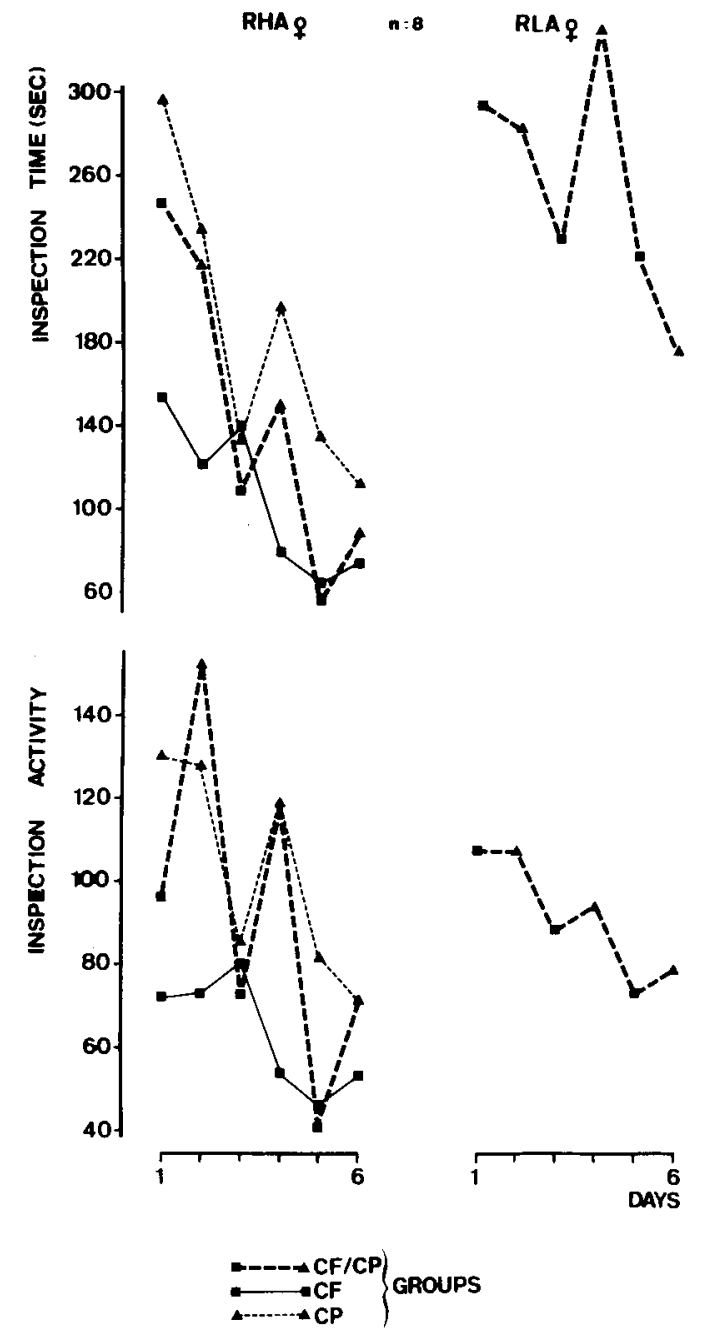

Figure 6. Comparison of all groups with regard to the patrolling behavior (inspection time, inspection activity) over the 6 testing days.

dition, but continuously increased thereafter and finally reached the same level as that for the CF mazes. Strain differences were seen for the CP conditions, but not for the CF conditions. There was a smaller CP/CF difference in the RLA than in the RHA CP/CF group. Higher choice frequencies were found for the bifurcated than for all other side alleys with all four groups.

\section{Discussion}

The results of this second experiment demonstrated pronounced differences between the centrifugal (CF) and centripetal (CP) mazes and between the two strains of rats used. In addition, a positive transfer from one maze to another was shown for inspection performance by the CP/CF groups of both strains.

The amount of activity was only marginally influenced by the two mazes. Activity increased with all three RHA groups, as it did in the first experiment with the RHA females, and remained at a constantly low level with the RLA females, as it did in a earlier experiment (Bättig et al., 1976). The alternate use of the $\mathrm{CP}$ and $\mathrm{CF}$ mazes slightly increased the activity of the RHA group on all CP as compared to CF days. No such oscillation was obtained in the RLA CP/CF group, nor was an analogous difference obtained between the two groups tested with the CP or CF maze alone. Therefore, it can be assumed that the increase in alley length from the $\mathrm{CF}$ to the $\mathrm{CP}$ maze was too small to reproduce reliably the activity increase obtained upon increase of alley length in the first experiment of this study, and that a slight difference in structural maze complexity had an additional masking effect on the expected activity increase (Uster et al., 1976). In contrast to the effects on activity, the effects on the number of $U$ turns and the resulting locomotor complexity were more pronounced. $U$ turns were-considerably more frequent with the $\mathrm{CP}$ than with the $\mathrm{CF}$ group, and showed pronounced oscillations with the $\mathrm{CP} / \mathrm{CF}$ groups of both strains. A recent extensive comparison between 12 maze arrangements with the same technique (Uster, 1977) indicated that this difference was probably not due to the CP/CF dimension of maze geometry. In that study, it was shown that the number of $U$ turns was consistently greater with the longer straight alley sections of the outer hexagonal alley than for the shorter corresponding sections of the inner hexagonal alley. On the basis of this assumption, it could also be speculated that a given length of straight sections works out to be "longer" for the RLA than for the RHA rats, because the oscillations of locomotor complexity were far greater in the RLA CP/CF group. However, additional experimentation will be needed to offer a satisfactory and consistent explanation for this phenomenon of locomotor complexity.

The efficiency of maze inspection has been affected by maze conditions and strain in the expected directon. It took the CP groups generally more time and more activity than the CF groups to enter eacn accessible part of the maze, because they had to overcome the innate centrifugal swing tendency (Uster et al., 1976). Therefore, the RHA CP/CF group showed more oscillation of the scores from their CF to their CP days than did the RLA CP/CF group, which reached the same overall efficiency as the RHA CP/CF group in terms of inspection activity but remained inferior in terms of inspection time due to their low level of locomotor activity. Although positive transfer was evident from the CP/CF conditions with both strains, the RLA strain appears to be superior to the RHA strain in the light of their smaller oscillation between the two mazes. 
The present results added additional evidence for a dissociation of maze ambulation into exploratory locomotor activity and exploratory learning processes: The two processes are differentially affected not only by strain and sex, but also by drugs (Bättig \& Schlatter, 1978). This latent learning process develops rapidly, particularly in view of the absence of any apparent consummatory or agonistic incentives. In addition, such latent learning in a maze readily transfers from one maze condition to another.

\section{REFERENCES}

Barnett, S. A., \& Cowan, P. E. Activity exploration, curiosity and fear: An ethological study. Interdisciplinary Science Reviews, 1976, 1, 43-62.

BätTIG, K., \& SCHLATTER, J. Effects of nicotine and amphetamine on maze exploration and on spatial memory by Roman high avoidance and Roman low avoidance rats. In K. Bättig (Ed.), Behavioral effects of nicotine. Basel: Karger, 1978.

Bättig, K., Driscoll, P., Schlatter, J., \& Uster, H. J. Effects of nicotine on the exploratory locomotion patterns of female Roman low- and high-avoidance rats. Pharmacology, Biochemistry and Behavior, 1976, 4, 435-439.

BERLYNE, D. E. Novelty and curiosity as determinants of exploratory behavior. British Journal of Psychology, 1950, 41, 68-80.

BERLYNE, D. E. The arousal and satiation of perceptual curiosity in the rat. Journal of Comparative and Physiological Psychology, 1955, 48, 238-246.

Berlyne, D. E. Conflict, arousal and curiosity. New York: McGraw-Hill, 1960.

Bindra, D., \& Spinner, N. Response to different degrees of novelty. Journal of the Experimental Analysis of Behavior, 1958, $1,341-350$.
Corman, D. C., \& Shafer, J. N. Open-field activity and exploratory behavior. Psychonomic Science, 1968, 13, 55.56.

Dember, W. N. Alternation behavior. In D. W. Fiske \& S. R. Maddi (Eds.), Functions of varied experience. Homewood, Ill: Dorsey Press, 1961.

Foshee, D. P., Vierck, Jr., C. J., Meier, G. W., \& Federspiel, C. Simultaneous measure of general activity and exploratory behavior. Perceptual and Motor Skills, 1965, 20, 445-451.

Kumar, R. Exploration and latent learning. Differential effects of dexamphetamine on components of exploratory behavior in rats. Psychopharmacologia (Berlin), 1969, 16, 54-72.

Le Yland, M., Robins, T., \& Iversen, S. D. Locomotor activity and exploration. The use of traditional manipulators to dissociate these two behaviors in the rat. Animal Learning \& Behavior, 1976, 4, 261.265.

Lore, R. K. Activity drive hypothesis. Psychological Bulletin, 1968, 70, 566-574.

Olton, D. S., \& Samuelson, R. J. Remembrance of places passed: Spatial memory in rats. Journal of Experimental Psychology: Animal Behavior Processes, 1976, 2, 97-116.

Robrins, T. W., \& IvErsen, S. D. A dissociation of the effects of d-amphetamine on locomotor activity and exploration in rats. Psychopharmacologia (Berlin), 1973, 28, 155-164.

Sachs, L. Angewandte Statistik (4. Auflage, Kap. 36.2), Berlin: Springer-Verlag, 1974.

Uster, H. J. Analyse des Explorationsverhaltens von Ratten in Labyrinthen unterschiedlicher Komplexität. Dissertation thesis No. 6004, Eidgenössische Technische Hochschule, Zürich, 1977.

Uster, H. J., Bättig, K., \& NÄgeli, H. H. Effects of maze geometry and experience on exploratory behavior in the rat. Animal Learning \& Behavior, 1976, 4, 84-88.

(Received for publication December 22, 1977; revision accepted April 15, 1978.) 